\title{
Derivatives of Erythropoietin That Are Tissue Protective But Not Erythropoietic
}

\author{
Marcel Leist, ${ }^{1 *}$ Pietro Ghezzi, ${ }^{2,3 *}$ Giovanni Grasso, ${ }^{3,4}$ \\ Roberto Bianchi, ${ }^{2}$ Pia Villa, ${ }^{2,5}$ Maddalena Fratelli, ${ }^{2}$ \\ Costanza Savino, ${ }^{2}$ Marina Bianchi, ${ }^{2}$ Jacob Nielsen, ${ }^{1}$ \\ Jens Gerwien, ${ }^{1}$ Pekka Kallunki, ${ }^{1}$ Anna Kirstine Larsen, ${ }^{1}$ \\ Lone Helboe, ${ }^{1}$ Søren Christensen, ${ }^{1}$ Lars O. Pedersen, ${ }^{1}$ \\ Mette Nielsen, ${ }^{1}$ Lars Torup, ${ }^{1}$ Thomas Sager, ${ }^{1}$ \\ Alessandra Sfacteria, ${ }^{3,4}$ Serhat Erbayraktar, ${ }^{3,6}$ \\ Zubeyde Erbayraktar, ${ }^{3,6}$ Necati Gokmen, ${ }^{6}$ Osman Yilmaz, ${ }^{3,6}$ \\ Carla Cerami-Hand, ${ }^{3,7}$ Qiao-wen Xie, ${ }^{3,7}$ Thomas Coleman, ${ }^{3,7}$ \\ Anthony Cerami, ${ }^{3,7} \uparrow$ Michael Brines $^{3,7}$
}

\begin{abstract}
Erythropoietin (EPO) is both hematopoietic and tissue protective, putatively through interaction with different receptors. We generated receptor subtypeselective ligands allowing the separation of EPO's bioactivities at the cellular level and in animals. Carbamylated EPO (CEPO) or certain EPO mutants did not bind to the classical EPO receptor (EPOR) and did not show any hematopoietic activity in human cell signaling assays or upon chronic dosing in different animal species. Nevertheless, CEPO and various nonhematopoietic mutants were cytoprotective in vitro and conferred neuroprotection against stroke, spinal cord compression, diabetic neuropathy, and experimental autoimmune encephalomyelitis at a potency and efficacy comparable to EPO.
\end{abstract}

Erythropoietin (EPO) is a pleiotropic cytokine originally identified for its role in erythropoiesis (1). Its hematopoietic effects on the bone marrow are mediated by the homodimeric erythropoietin receptor $\left[(\mathrm{EPOR})_{2}\right]$, a class 1 cytokine receptor. Sasaki and others identified the production of EPO in the central nervous system and, later, its neuroprotective function (1-7). Eventually, a proof-of-concept for neuroprotection by peripherally dosed EPO was obtained in a phase II clinical trial in cerebral ischemia (8).
Desialylated EPO (asialoEPO) has the same $(\mathrm{EPOR})_{2}$ affinity and neuroprotective properties as EPO, but an extremely short plasma half-life. Both molecules share the capacity of crossing an intact blood-brain barrier when dosed peripherally (3, 8-10), but bear also the risk of unwanted effects linked to the chronic overstimulation of $(\mathrm{EPOR})_{2}$, for example, on the bone marrow.

Extensive structure-activity relationship (SAR) studies of EPO have identified regions and amino acids essential for binding to $(\text { EPOR })_{2}(11)$, and many chemical modifications that abolish EPO's hematopoietic bioactivity are known $(12,13)$. However, the receptor complex mediating the neuroprotective effects of EPO differs from the hematopoietic receptor with respect to apparent affinity for EPO, apparent molecular weight, and associated proteins (2). The EPO receptor has been reported to associate functionally with other cytokine receptors such as CD131 $(14,15)$, and a region of EPO not within the (EPOR) 2 binding domains has been associated with neuroprotective effects (16). On the basis of these observations, we postulated that molecular changes to erythropoietin that neutralize erythropoiesis would not necessarily alter tissue-protective potency. One known modification silencing erythropoiesis is the carbamylation of lysines, a process well recognized to profoundly alter protein conformation and function. Surprisingly, we found that carbamylated EPO is neuroprotective and therefore introduces a new class of neuroprotective cytokines that lack erythropoietic activity yet engage a tissue-protective receptor.

All lysines in EPO were transformed to homocitrulline by carbamylation (17) (fig. S1A). CEPO, the resultant product, completely lacked bioactivity in the in vitro UT7 hematopoiesis bioassay (Table 1) and failed to bind to EPOR on these cells (Fig. 1A). However,

${ }^{1} \mathrm{H}$. Lundbeck A/S, 2500 Valby, Denmark. ${ }^{2}$ Mario Negri Institute of Pharmacological Research, 20157 Milano, Italy. ${ }^{3}$ The Kenneth S. Warren Institute, Ossining, NY 10562, USA. ${ }^{4}$ University of Messina, 98122 Messina, Italy. ${ }^{5}$ Consiglio Nazionale delle Ricerche, Institute of Neuroscience, 20129 Milano, Italy. ${ }^{6}$ Dokuz Eylul University School of Medicine, Izmir 35340, Turkey. ${ }^{7}$ Warren Pharmaceuticals, Inc., Ossining, NY 10562, USA.

*These authors contributed equally to this study. †To whom correspondence should be addressed. Email: acerami@kswi.org 
CEPO protected P19 cells from apoptosis (Table 1) and prevented $N$-methyl-D-aspartate (NMDA)-induced apoptosis of hippocampal cells with a potency $\left(\mathrm{IC}_{50}\right.$ of 6 to $\left.10 \mathrm{pM}\right)$ and efficacy (50 to $80 \%$ specific protection) similar to EPO (Fig. 1B). Thus, CEPO represents the prototype for a family of designer cytokines with a novel activity spectrum.

We further explored the new principle that erythropoietic bioactivity and cytoprotection followed different SARs, generating mutants of EPO known to disrupt erythropoietic activity, but not conformation $(18,19)$. Some mutants, such as EPO-S100E or EPO-R103E, retained a high cytoprotective efficacy despite drastically reduced (EPOR) $)_{2}$ affinity (Table 1 ). Thus, the tissue-protective potential of EPO could be separated from $(\mathrm{EPOR})_{2}$ interaction by multiple approaches affecting the protein backbone of

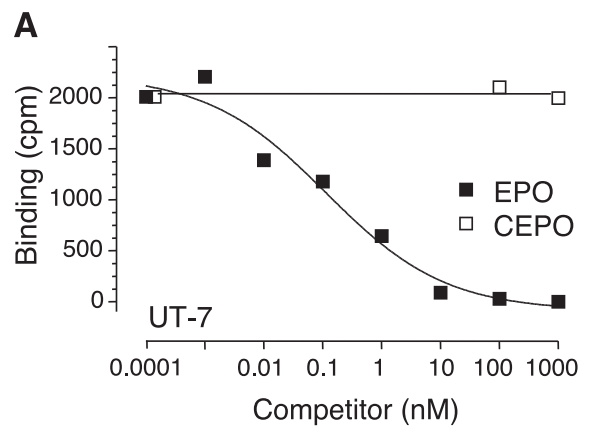

EPO, whereas changes in sialylation had no effect on the receptor affinities and in vitro bioactivities of the different types of ligands (Table 1). We further characterized the binding of CEPO to live cells $(\mathrm{CHO}, \mathrm{BaF} / 3)$ overexpressing $(\mathrm{EPOR})_{2}(17)$. In several of these experiments, asialoCEPO was used to exclude any binding effects of sialic acid-specific lectins previously described for neural cells (20). Depending on the cellular context and receptor species (apparent affinity of human EPO for huEPOR was about three times as high as that for rodent EPOR), EPO bound at 10 to $200 \mathrm{pM}$, while CEPO or asialoCEPO did not bind at concentrations up to 10,000 pM (fig. S2, A and B, and Fig. 1A).

In contrast to the above hematopoiesis model systems, we found that EPO and asialoCEPO had similar binding affinities for living PC-12 cells as representative of a neural cell type (Fig.

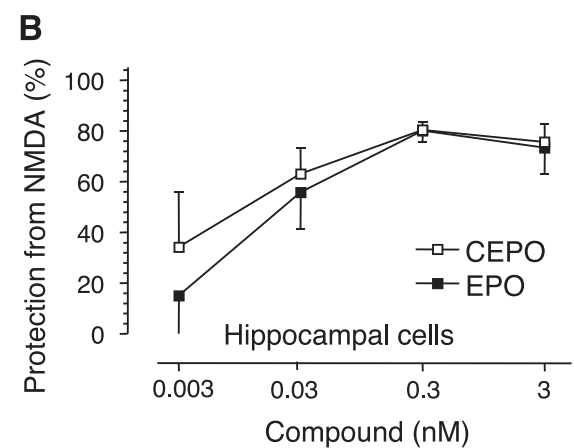

Fig. 1. CEPO is neuroprotective without binding to EPO-R. (A) Binding competition with iodinated EPO was determined for UT7 cells. Cells were incubated with radiotracer and graded doses of unlabeled EPO or CEPO and analyzed for radiotracer binding. (B) CEPO/EPO was added to hippocampal neurons 24 hours before challenge with NMDA, and toxicity was evaluated 24 hours later by counting apoptotic neurons.

A

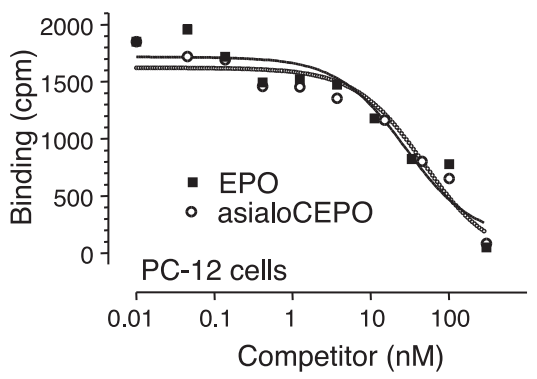

Fig. 2. Differential binding and erythropoietic signaling of EPO, CEPO, and asialoCEPO. (A) PC-12 (neural-type) cells were incubated with radiotracer (EPO) and graded doses of unlabeled EPO or asialoCEPO and analyzed for radiotracer binding. Data are in counts per minute (cpm) for quadruplicate samples. (B) BaF/3-EPOR cells were stimulated for 10 min with CEPO or EPO. Activation was analyzed by Western blotting of cell lysates with phosphorylation site-specific antibodies (PYJak2; Stat5). Equal loading was confirmed by reprobing of membranes against nonphosphorylated Jak2. (C) CEPO was intravenously in-

B

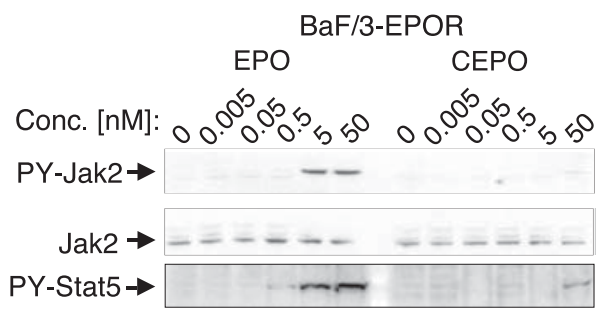

C

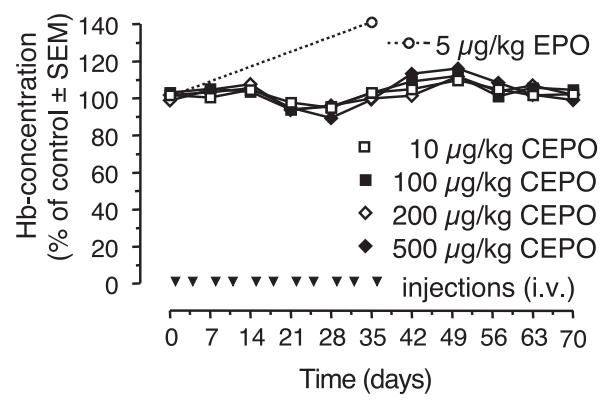

jected (i.v.) biweekly to mice $(n=8)$. Injection days are marked by triangles. Data are expressed as percentage of saline-treated animals [control hemoglobin $(\mathrm{Hb})=15 \pm 3 \mathrm{mg} / 100 \mathrm{ml}$ ]. One group of mice was injected with $5 \mu \mathrm{g} / \mathrm{kg} \mathrm{EPO}$, and Hb levels were determined after 5 weeks as positive control.
2A). These data suggest that while CEPO does not interact with the classical (EPOR $)_{2}$ to a degree detectable by radioligand binding assays, EPO and CEPO appear to compete for a common binding site on some (neural) cell types. We assessed potential interaction of CEPO with the (EPOR) 2 by measuring the phosphorylation of the transcription factor STAT-5 or of Jak2, a downstream kinase directly activated upon ligand binding to the EPOR. In $\mathrm{BaF} / 3-\mathrm{EPOR}$ cells, EPO concentrations $>0.5 \mathrm{nM}$ stimulated the phosphorylation of signaling molecules. In contrast, CEPO concentrations up to $50 \mathrm{nM}$ showed only minimal activity (Fig. 2B).

In clinical applications of neuroprotection, the ability of the engineered cytokines to cross the blood-brain barrier is critical. We found that CEPO distributed at least as well into the cerebrospinal fluid (CSF) as did EPO. For example, after intravenous (iv) bolus CEPO injection ( rat, $44 \mu \mathrm{g} / \mathrm{kg} ; n=6$ ), pM concentrations ( $15 \pm 6 \mathrm{pM} \mathrm{CEPO} \mathrm{versus}$ $7 \pm 2$ pM EPO) were detected in CSF after 4 hours, and after 24 hours the CSF CEPO concentrations were still significantly elevated above baseline $(4 \pm 2 \mathrm{pM})$. The plasma pharmacokinetic parameters for CEPO were also in the same range as those for EPO. The plasma elimination half-life (rat, $44 \mu \mathrm{g} / \mathrm{kg}$ iv; $n=4$ ) was 3.3 hours. Peak concentrations of $150 \mathrm{pM}$ were reached 14 hours after subcutaneous injection (rat, $44 \mu \mathrm{g} / \mathrm{kg} ; n=4$ ), and the plasma concentrations remained significantly elevated above $2 \mathrm{pM}$ (the erythropoietic threshold for EPO) for $>20$ hours (17).

CEPO circulates long enough in vivo to potentially trigger hematopoiesis independently of a classical (EPOR) ${ }_{2}$ interaction. Hence, we examined this possibility in several systems. Biweekly injections of up to $500 \mu \mathrm{g} / \mathrm{kg}$ CEPO did not increase the hematocrit in mice (not iron supplemented), in contrast with identically dosed $5 \mu \mathrm{g} / \mathrm{kg}$ EPO (Fig. 2C). Even five times weekly injections of CEPO administered subcutaneously did not increase the hematocrit. Moreover, CEPO was tested on rats in which an 8-week daily dosing of $50 \mu \mathrm{g} / \mathrm{kg}$ had no augmenting effect on hemoglobin levels or hematocrit. Finally, mice were dosed subcutaneously daily for 4 weeks with CEPO at 10,50 , or $200 \mu \mathrm{g} / \mathrm{kg}$. Whereas the control group receiving EPO (10 $\mu \mathrm{g} / \mathrm{kg}$ ) showed a 1.7 -fold hematocrit increase, none of the CEPO groups showed any increase after 4 weeks or after a recovery period of an additional 2 weeks $(<3 \%$ increase in any group). Thus, CEPO is not erythropoietic even when continuously present at high concentrations in plasma.

We also determined whether the modified EPOs might function as antagonists to the $(\mathrm{EPOR})_{2}$. The new variants were completely devoid of competitive effects, even at concentrations up to 300 times as high as those of EPO (Table 1 and fig. S2C). 
In a cerebral infarct model, CEPO showed the same degree of tissue protection as reported for EPO and was effective over the same dose range ( 5 to $50 \mu \mathrm{g} / \mathrm{kg}$ ). Delayed administration of CEPO by up to 4 hours after occlusion of the middle cerebral artery still conferred protection (Fig. 3A). This broad therapeutic time window has also been reported for EPO $(9,10)$ and distinguishes CEPO as a potential stroke therapeutic different from many other compounds that failed in clinical trials. Moreover, tissue protection by CEPO in the stroke model correlated well with a reduced inflammatory response in the ipsilateral hemisphere: Interleukin-6 levels were significantly $(P<0.05)$ reduced from $11,000 \mathrm{U} / \mathrm{g}$ in the control group to $5600 \mathrm{U} / \mathrm{g}$ in the CEPO group, and monocyte chemoattractant protein-1 levels from $3150 \mathrm{pg} / \mathrm{g}$ to $1720 \mathrm{pg} / \mathrm{g}(P=0.03)$. In addition, we evaluated CEPO's effects on loss of function resulting from retinal ischemia (not shown) or sciatic nerve compression (Fig. 3B) and, again, observed a protective effect to the same degree as that described for EPO $(9,10)$.

Next, CEPO was tested in an established model of spinal cord injury (9). Neurological function after injury was significantly improved in the animals treated over 6 weeks with CEPO compared with the saline or EPO groups (Fig. $3 C)$. A delay of 24 hours in treatment resulted in nearly identical recovery as that observed for animals receiving CEPO immediately after injury (Fig. 3D). Even when the first dose was given with a delay of 48 hours (not shown) or 72 hours after injury, we still observed a significant beneficial effect on the neurological function (Fig. 3D).

EPO is beneficial in another subchronic disease model, experimental autoimmune encephalomyelitis (EAE) (3). The neurological deficits in mice that had been immunized with myelin oligodendrocyte glycoprotein (MOG) to induce EAE were improved by CEPO over a prolonged observation period (Fig. 3E). In a modified setup of the model, first a stable disease state was triggered in the animals. Even 4 weeks after the robust plateau of neurological dysfunction was reached, a three-times-per-week treatment with CEPO significantly improved neurological function (Fig. 3E). Finally, we observed that CEPO reproduced the recently discovered beneficial effects of EPO (21) on diabetic peripheral neuropathy (Fig. 3F) (17).

We have presented the rationale, synthesis, and characterization of CEPO, an engineered cytokine with an activity spectrum preferentially targeting tissues outside the bone marrow. The findings have implications both for the biology of EPO and for the design of new tissue-protective therapies. Unlike other modified cytokines entering the clinic, the new class of compounds is not based on alterations of stability, plasma half-life, or antigenicity with an otherwise similar bioactivity spectrum as the parent cytokine. On the contrary, the pharma- cokinetic properties of CEPO are similar to EPO's, while the pharmacodynamics exhibit an unprecedented profile. CEPO exhibits a new mode of action, best explained by engaging an alternative receptor signaling tissue protection. In this respect, CEPO differs strikingly from asialoEPO, which behaves on the molecular level exactly as EPO (9).

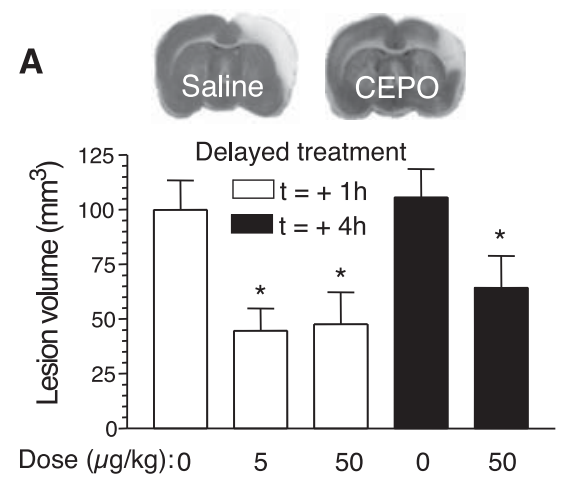

C

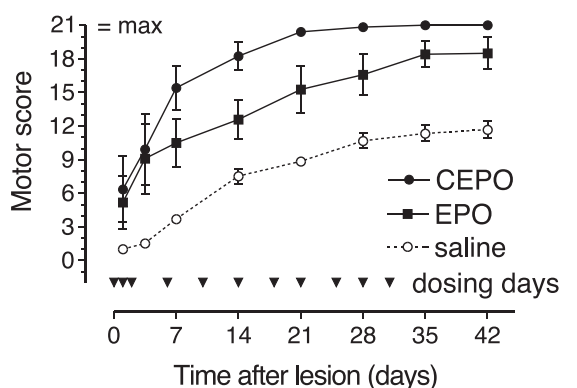

E

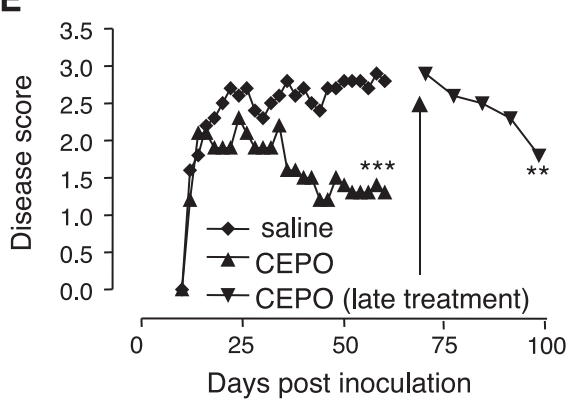

\section{$\mathbf{F}$}

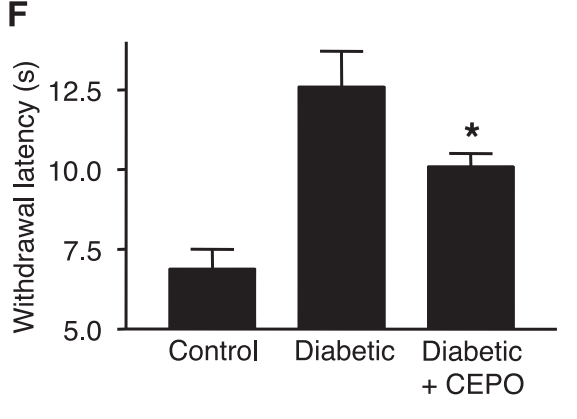

Fig. 3. Neuroprotection by CEPO. (A) Single intravenous injections of CEPO were given 1 hour or 4 hours after occlusion in a rat model of focal ischemia. Reperfusion was initiated after $60 \mathrm{~min}$, and the infarct volume was measured after 24 hours. Data are means + SD; $n=8$. (Insert) Representative images of tetraphenyl tetrazolium chloride-stained sections (at $2 \mathrm{~mm}$ caudal to bregma) showing infarct size. (B) The sciatic nerve of rats $(n=6)$ was compressed for 1 min and CEPO or vehicle was dosed immediately after the crush as a single intravenous bolus. The static sciatic index (SSI) is an indicator of motor function after sciatic nerve injury, and data are expressed as percentage versus saline-treated animals. (C) EPO, CEPO, or saline was injected intravenously immediately after surgical induction of spinal cord injury and over a period of 6 weeks, as indicated by triangles. Motor function of all animals was evaluated for 42 days, and data are presented as means \pm SEM; $n=6$. (D) The experiment was performed as in (C), but the dosing of CEPO (10 $\mu \mathrm{g} / \mathrm{kg}$ ) was delayed by 0,24 , or 72 hours after the spinal cord compression. Injection days are marked by triangles. Data are means $\pm \mathrm{SEM} ; n=6$. (E) Chronic EAE was induced by MOG; $n=8$. The motor deficit was scored on a scale of 0 to 5 . Animals were treated for 8 weeks with CEPO $(50$ $\mu \mathrm{g} / \mathrm{kg}$; three times weekly) versus saline. Alternatively, they were left untreated for 10 weeks and then dosed for 4 weeks with CEPO as above. (F) Neuropathy in streptozotocin-treated rats. Four weeks after induction of the diabetic state, the thermal nociceptive threshold was quantified by measurement of the time to paw withdrawal in a "hot plate" test. CEPO $(50 \mu \mathrm{g} / \mathrm{kg})$ was injected subcutaneously three times per week for four weeks. ${ }^{*} P<0.05$; ${ }^{* *} P<0.01 ;{ }^{* * *} P<0.001$. 
Table 1. Comparison of EPOR binding, as well as hematopoietic and neuroprotective properties of different variants of EPO. EPOR binding experiments were performed on dimerized Fc-fusion proteins and measured as inhibition of EPO binding. Block of UT7 proliferation was measured in the presence of 50 PM EPO and 0.3 to $30 \mathrm{nM}$ compound. No, less than $10 \%$ block; n.d., not determined; neurons, rat hippocampal neurons exposed to NMDA; P19, murine teratocarcinoma cells stressed by serum withdrawal.

\begin{tabular}{lccccc}
\hline Modification & $\begin{array}{c}\text { EPOR } \\
\mathrm{IC}_{50}\end{array}$ & $\begin{array}{c}\text { UT7 } \\
\text { (pM) }\end{array}$ & $\begin{array}{c}\text { ET7 } \\
(\mathrm{pM})\end{array}$ & block & (\% protection \pm SD at $300 \mathrm{pM})$ \\
\hline Wild-type EPO & 10 & $10-30$ & n.d. & $78 \pm 13$ & $49 \pm 12$ \\
AsialoEPO & 14 & $10-30$ & n.d. & $71 \pm 15$ & n.d. \\
CEPO & $>10,000$ & $>10,000$ & No & $70 \pm 9$ & $49 \pm 10$ \\
AsialoCEPO & $>10,000$ & $>10,000$ & No & $69 \pm 16$ & n.d. \\
S100E-EPO & 100 & $>10,000$ & No & $66 \pm 9$ & $55 \pm 15$ \\
R103E-EPO & $>10,000$ & $>10,000$ & No & $55 \pm 13$ & n.d. \\
\hline
\end{tabular}

observations that EPO receptor expression in tissues correlates with the protective effect of EPO $(10,22)$. Such heteromeric receptors would likely present new binding sites and therefore new pharmacological characteristics.

Although the precise means by which tissue-protective cytokines signal remain to be clarified, the availability of compounds such as CEPO that do not trigger (EPOR) also opens possibilities to distinguish experimentally between EPO's tissue-protective effects (e.g., antiapoptosis) and its potentially detrimental effects [e.g., procoagulant and prothrombotic effects (23) within the microvasculature] and excessive erythropoiesis upon chronic dosing. With these compounds, it is now possible to trigger EPO-mediated

1. R. Sasaki, Intern. Med. 42, 142 (2003).
12. K. C. Mun, T. A. Golper, Blood Purif. 18, 13 (2000).

13. R. Satake, H. Kozutsumi, M. Takeuchi, K. Asano, Biochim. Biophys. Acta 1038, 125 (1990).

14. Y. Hanazono, K. Sasaki, H. Nitta, Y. Yazaki, H. Hirai, Biochem. Biophys. Res. Commun. 208, 1060 (1995).

15. P. T. Jublinsky, O. I. Krijanovski, D. G. Nathan, J. Tavernier, C. A. Sieff, Blood 90, 1867 (1997).

16. W. M. Campana, R. Misasi, J. S. O’Brien, Int. J. Mol. Med. 1, 235 (1998).

17. Materials and methods are available as supporting material on Science Online.

18. J. Grodberg, K. L. Davis, A. J. Sytkowski, Arch. Biochem. Biophys. 333, 427 (1996).

19. J. P. Boissel, W. R. Lee, S. R. Presnell, F. E. Cohen, H. F. Bunn, J. Biol. Chem. 268, 15983 (1993).

20. G. M. Yousef, M. H. Ordon, G. Foussias, E. P. Diamandis, Biochem. Biophys. Res. Commun. 284, 900 (2001).

21. R. Bianchi et al., Proc. Natl. Acad. Sci. U.S.A. 101, 823 (2004).

22. T. Eid, M. Brines, Clin. Breast Cancer 3 (suppl. 3), S109 (2002).

tissue-protective pathways without cross-talk with the hematopoietic system.

\section{References and Notes}

2. S. Masuda et al., J. Biol. Chem. 268, 11208 (1993).

3. M. L. Brines et al., Proc. Natl. Acad. Sci. U.S.A. 97 10526 (2000).

4. Y. Konishi, D. H. Chui, H. Hirose, T. Kunishita, T. Tabira, Brain Res. 609, 29 (1993).

5. M. Sakanaka et al., Proc. Natl. Acad. Sci. U.S.A. 95, 4635 (1998).

6. M. Digicaylioglu, S. A. Lipton, Nature 412, 641 (2001).

7. A. L. Siren et al., Proc. Natl. Acad. Sci. U.S.A. 98, 4044 (2001).

8. H. Ehrenreich et al., Mol. Med. 8, 495 (2002).

9. S. Erbayraktar et al., Proc. Natl. Acad. Sci. U.S.A. 100, 6741 (2003).

10. H. Ehrenreich et al., Mol. Psychiatry 9, 42 (2004).

11. J. Grodberg, K. L. Davis, A. J. Sykowski, Eur. J. Biochem. 218, 597 (1993) 